\title{
IMPROVING THE INTERNAL ACCURACY OF UAV-IMAGE BLOCKS USING LOCAL LOW-ALTITUDE FLIGHTS AND SCALE-BARS
}

\author{
M. Maboudi*, A. Elbillehy, Y. Ghassoun, M. Gerke \\ Institute of Geodesy and Photogrammetry, Technische Universität Braunschweig, Germany \\ (m.maboudi, a.elbillehy, y.ghassoun, m.gerke)@tu-bs.de
}

\section{Commission ICWG I/II}

KEY WORDS: UAV mapping, local measurement, photogrammetric block, internal accuracy, calibrated scale-bar

\begin{abstract}
:
Accurate image-based measurement based on UAV data is attracting attention in various applications. While the external accuracy of the UAV image blocks could be mainly affected by object-space information like number and distribution of ground control points and RTK-GNSS accuracy, its internal accuracy highly depends on camera specifications, flight height, data capturing setup and accuracy of scale estimation. For many small-scale projects accurate local measurements are highly demanded. This necessitates high internal accuracy of images block which could be transferred from model space to object space by accurate estimation of the scale parameter. This research aims at improving the internal accuracy of UAV image blocks using low-altitude flight(s) over small parts of the project area without using any ground control points. Possible further improvement by using calibrated scale-bars which serve as scale-constraints is also investigated. To this end, different scenarios of the flight configuration and distance measurements in the two photogrammetric blocks are also considered and the results are analyzed. Our investigations show 50\% accuracy improvement achieved by performing local flights over small parts of the scene, given that RTK information is available. Moreover, adding accurate scale-bars increased the accuracy improvement to $67 \%$. Furthermore, when RTK information is not available, adding local low-altitude flights and scale-bars decrease the error of local distance measurement form 1-3 meters to less than 4 centimeters.
\end{abstract}

\section{INTRODUCTION}

Nowadays drones are getting more and more popular for many applications like high precision mapping and $3 \mathrm{D}$ reconstruction to name a few (Gerke and Przybilla, 2016; Kraft et al., 2016; Malihi et al., 2018; Martínez-Carricondo et al., 2018; Sahebdivani et al., 2020). Hence, analyzing the internal and external accuracy of drone image blocks is very important. Many researchers also investigate the effects of different imaging parameters and flight settings on the expected accuracy (Ekaso et al., 2020; Ferrer-González et al., 2020; Gerke and Przybilla, 2016).

The precision of measuring coordinates of a point on a digital image is 0.3 to 0.5 of the pixel size, as a rule of thumb. Given that the geometry of stereo photography is ideal (high $\mathrm{B} / \mathrm{H}$ ratio) this number could be transferred to the model space and the precision of computed coordinates in the model space (internal accuracy) could be around 0.5 pixel in the model scale. So, provided that the scale parameter is solved perfectly, the accuracy could be 0.5 GSD (Ground Sampling Distance) on the ground. Theoretically, in order to solve the scale, at least two horizontal ground control points (GCPs) or one known distance in the object space is necessary. Practically, number and distribution of GCPs (Gerke and Przybilla, 2016) and accuracy of the known distance has a critical effect on the final accuracy. Scaling up the problem from two images to a photogrammetric block, accumulative errors cause block deformation and the internal accuracy of the block may be degraded, considerably. In order to avoid this problem and also georeferencing the image block, images should be captured using a drone which is equipped with RTK GNSS or adequate and well distributed full GCPs in object space should be measured.

On the one hand using just GNSS information the expected accuracy (both internal and external) will not be better than the accuracy of GNSS measurements (currently 2-3 cm for RTK GNSS). On the other hand, number and distribution of precise GCPs play the most important role on the expected accuracy. However due to different errors in estimation of the scale parameter, the horizontal accuracy would be in the range of 1 GSD and the vertical accuracy would be around 1-2 GSD for usual UAV projects.

In many projects, local measurements are desired and internal accuracy of the results should be much better than $2-3 \mathrm{~cm}$. However, just using GNSS information and even with high quality cameras this goal could not be accomplished. Hence using an accurate surveying network for providing accurate GCPs is necessary. Though, providing many accurate and welldistributed GCPs needs a lot of time, effort and is costly. This motivated this research to investigate the feasibility of using low altitude local flights and accurate scale-bars for improving the internal accuracy of the photogrammetric block without using any GCPs. Utilizing low altitude local flights and using scalebars instead of GCPs may highly improve the time and cost competence of the UAV-based projects for local measurement which do not need geo-referenced information. In this regard, different scenarios of the flight configuration and distance measurements in the photogrammetric block are considered and the results are analyzed thoroughly.

\footnotetext{
${ }^{*}$ Corresponding author
} 


\section{RELATED WORKS}

As it has been mentioned previously, many researchers studied the impact of different parameters on the final accuracy. These parameters can be summarized by 1) the limitations regarding the flight time of the drones. Especially, if a large area should be captured in a short time, flights must be performed at higher altitude, which decrease the special resolution (González et al., 2019). 2) The limitation regarding flight altitude and the determined ground sample distance in a specific area, especially within cities and industrial and construction areas (Burdziakowski, 2020). 3) The selection of the camera mounted on UAV is the most important factor regarding the results to be achieved (Przybilla et al., 2019). 4) RTK and GNSS used 5) Number and distribution of GCPs that are distributed and their measurements accuracy(Sanz-Ablanedo et al., 2018).

In this context, to improve the accuracy (Kedzierski and Wierzbicki, 2016) applied filtration in frequency domain to improve the image radiometry and hence the reflection from objects in the images and reduce the impact of poor lighting on local contrast. The algorithms decreased the error of the digital aerial triangulation by $10 \%$ and increased the number of tie points by $15 \%$. (Burdziakowski, 2020) and (González et al., 2019) have used Super resolution (SR) algorithms to improve the quality of the original images and the geometric and interpretative quality of the final photogrammetric product. In another study, the geometric quality of different cameras was investigated, especially the parameters that can influence the geometric quality (Przybilla et al., 2019). Three UAV systems (Zenmuse X4S (20 Mpix) and X5S (20.8 Mpix), as well as a Phase One IXU 1000 (100 Mpix)) and 45 GCPs were utilized. The results show that the cross flights have a positive effect and the distribution of GCPs has a major effect on the bundle block adjustment quality. A full automated workflow is introduced in (Ghassoun et al., 2021) to extract the railway track with sub-millimeter accuracy. By using a PhaseOne iXM 100MP camera to capture images on $35 \mathrm{~m}$ height and coded targets as GCPs, the nominal GSD that can be achieved with the selected system is $0.9 \mathrm{~mm}$. First, preliminary results show that fully automatic data flow from the image acquisition to the determination of the rail position is possible. Also, scene constraints are used (Gerke and Nyaruhuma, 2009) in the block adjustment to optimize the exterior orientation and to calibrate the interior orientation and distortion parameters..

The accuracy of the drone based volumetric surveys using SfM (Structure from Motion) software in comparison to the traditional survey methods is investigated in (Adjidjonu and Burgett, 2021). The authors used two pyramids with different volumes to mimic stockpile of earth on construction site. Then, nadir and oblique images were captured during flights with different altitudes (40ft, $125 \mathrm{ft}, 350 \mathrm{ft})$. The results have shown that nadir and oblique images at $125 \mathrm{ft}$ have the most consistent results, with error value ranging from $0.35 \%$ to $3.01 \%$ from the actual volume for both pyramids, respectively. Also, (Manfreda et al., 2019) studied the influence of the flight mode and its configurations and the influence of the distribution of the GCPs on the production of high-quality topographic models. For this purpose, six flight scenarios with different configurations such as changing flight trajectories, camera tilt, and the elevation of the flight were planned and 16 GCPs were distributed. The study has shown that the combination of different flights (nadir and $20^{\circ}$ tilted camera) leads to higher accuracy, especially for reducing the vertical error. Also, the flights operated on two orthogonal routes lead to minimize the error.

\section{METHOD AND EXPERIMENTS}

As discussed in section 1, GSD of the images and the accurate estimation of scale parameter are two key parameters for determination of the accuracy of the local measurements (length, volume, ...) based on photogrammetric blocks. Having accurate and well-distributed GCPs causes a high cost, time, and effort. Therefore, in our experiments, we try to test a faster and much cheaper solution for that, namely using low altitude local flights in small parts of the project and employing calibrated surveying scale-bars. GSD of the images could also be affected by the camera which is used in the flight missions and also by the flight height. Without changing the camera, flight height then would be very critical for getting smaller GSD. However, in practice, flying in the low constant altitude over the whole scene is not always possible. One common reason is the existence of tall manmade objects or trees, and in addition for larger areas this would lead to a tremendous number of images. These common restrictions in practical projects, was the motivation for employing low-altitude flights above smaller regions as well as employing accurate scale-bars to increase the internal accuracy of a photogrammetric block for local measurements. Therefore, the main objective of our experiments concerns two main questions: a) Do the low altitude local flight(s) improve the internal accuracy of a UAV image block? b) How does employing the calibrated scale-bars influence the accuracy of local measurements in a UAV project. To answer these questions, two experiments with some different setups are tested.

\subsection{Study area and hardware setup}

The study area is located in the north campus of the Technical University of Braunschweig, Germany and covers an area of $75 \times 75 \mathrm{~m}^{2}$ (Figure 1).

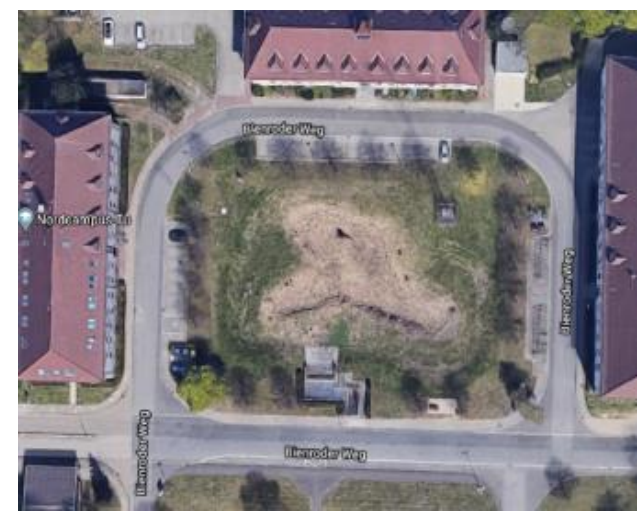

Figure 1: Study area

Existence of relatively tall buildings around this area and also a radio tower inside this study area makes flights in low altitude (less than 50 meters) impossible/unsafe. Images from this area are taken from a DJI Phantom 4 RTK drone equipped with an RGB camera FC6310R with resolution of $5472 \times 3648$, and the focal length of $8.580 \mathrm{~mm}$. The utilized scale-bars are classical levelling bars with photogrammetric coded targets at the both ends (Figure 2). The calibrated distance between coded targets on each scale-bar is measured using AICON DPA system with a precision of $50 \mu \mathrm{m}$. In the field we distributed points which were measured with a total station in a local area network survey.

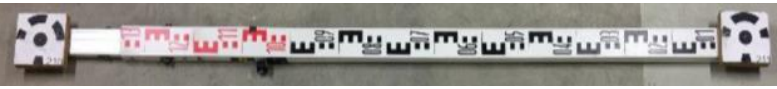

Figure 2: calibrated scale-bars 
Two experiments with different flight configurations and object space measurements are carried out as described below.

\subsection{First experiment}

In the first experiment, 3 scale-bars (Figure 3d) are distributed in each of 3 corner areas (Figure $3 a$ ) which serve as scale constraints in the block adjustment. The closed surveying network consists of $3 \mathrm{D}$ coordinates of four points in the corners of the area with a standard deviation in XYZ of $1 \mathrm{~mm}$. These points did not contribute in the block adjustment. We used these points as check points to compute the distance between them (Figure $3 b$ ) for evaluating the results. We refer to the length of the distance between each pair of check points as check distance.

One flight is performed at the height of $55 \mathrm{~m}(\mathrm{GSD}=1.6 \mathrm{~cm})$ over the whole area (Figure $3 \mathrm{c}$ ) and 3 flights at the height of $10 \mathrm{~m}$ over 3 corner areas $(\mathrm{GSD}=0.27 \mathrm{~cm})$. In this experiment random errors with $\mu=10 \mathrm{~m}$ and $\sigma=2 \mathrm{~m}$ are added to projection center coordinates to simulate the effect of losing/lacking RTK information in a UAV mapping project.

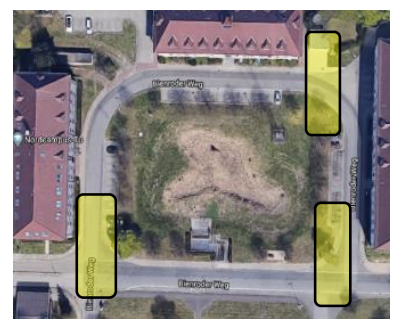

a

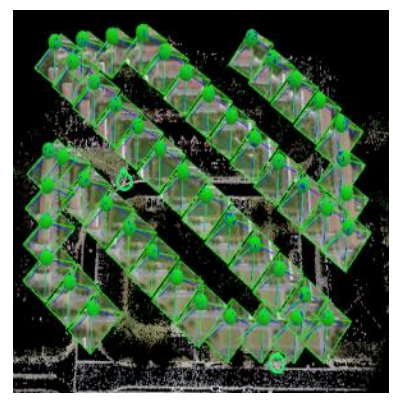

c

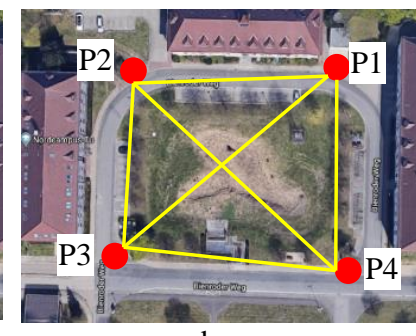

b

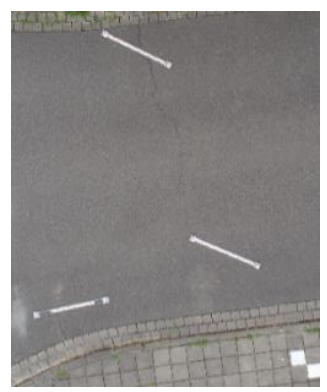

d
Figure 3: Configuration of the first experiment; a) Study area captured from $55 \mathrm{~m}$ height and 3 small areas captured from 10 $\mathrm{m}$ height, b) Check distances for quality inspection, c) $55 \mathrm{~m}$ images block, d) distributed calibrated scale-bars

The aim of this experiment is the investigation of the possibility of improving the accuracy of the local measurements using scalebars in the small area flown at low altitude without accurate projection center coordinates.

In this experiment 48 images are captured at $55 \mathrm{~m}$ altitude (Figure $3 \mathrm{c}$ ) and 94 images over 3 small corners at $10 \mathrm{~m}$ flight height. First just the images with projection center coordinates of around $10 \mathrm{~m}$ accuracy and without any other object space information are used (setup 1). We used Agisoft Metashape and Pix4DMapper and in both cases the accuracy of check distances are quite similar. Therefore, in the second experiment we just use Agisoft Metashape.

The length of 6 check distances as well as the corresponding lengths computed from UAV image block are listed in Table 1 where $\mathrm{D} i j$ indicates the distance between the points $\mathrm{P} i$ and $\mathrm{P} j$.

\begin{tabular}{|l|c|c|c|c|c|c|}
\hline & $\begin{array}{c}\text { D12 } \\
(\mathrm{m})\end{array}$ & $\begin{array}{c}\mathrm{D} 23 \\
(\mathrm{~m})\end{array}$ & $\begin{array}{c}\text { D34 } \\
(\mathrm{m})\end{array}$ & $\begin{array}{c}\text { D14 } \\
(\mathrm{m})\end{array}$ & $\begin{array}{c}\text { D13 } \\
(\mathrm{m})\end{array}$ & $\begin{array}{c}\text { D24 } \\
(\mathrm{m})\end{array}$ \\
\hline Ref. & 63.216 & 50.480 & 70.636 & 62.167 & 87.560 & 86.821 \\
\hline UAV & 61.709 & 49.275 & 66.696 & 60.652 & 85.439 & 84.724 \\
\hline error & 1.507 & 1.205 & 3.940 & 1.515 & 2.121 & 2.097 \\
\hline
\end{tabular}

Mean error (bias): $2.064 \mathrm{~m}$

RMSE: $2.252 \mathrm{~m}$

Standard deviation: $0.901 \mathrm{~m}$

Table 1. Check distances (images block with $55 \mathrm{~m}$ altitude)

The first row of Table 1 shows the names, second row contains reference lengths (surveying measurements), third row is the distances computed from UAV block with $55 \mathrm{~m}$ average altitude (without GCP and with projection center error around $10 \mathrm{~m}$ ) and the last row shows the error. The bias, RMSE and standard deviation of the errors are $2.064 \mathrm{~m}, 2.252 \mathrm{~m}$ and $0.901 \mathrm{~m}$, respectively. It could be understood from this result that using images with very low accurate camera positions $(10 \mathrm{~m} \pm 2 \mathrm{~m})$ and without GCPs leads to very low accurate $(2.06 \pm 0.9 \mathrm{~m})$ local distance measurements. One solution is to establish some welldistributed and accurate GCPs which is time consuming and costly. Another solution is to use RTK information from drone GNSS receiver which may not be available. Moreover, the final accuracy is still limited by RTK-GNSS accuracy level. This motivates our proposed method to use low altitude local flights and also some scale-bars in the scene.

By employing images from low altitude (10m) flights over 3 corners of the scene (Figure $3 \mathrm{a}$ ) we computed 3 small blocks and merged them to the main block (images with $55 \mathrm{~m}$ altitude) and readjusted the merged blocks (Setup 2). The results of this setup are listed in Table 2. The mean and standard deviation of the errors are $-0.024 \mathrm{~m}$ and $0.027 \mathrm{~m}$, respectively.

\begin{tabular}{|l|c|c|c|c|c|c|}
\hline & $\begin{array}{c}\text { D12 } \\
(\mathrm{m})\end{array}$ & $\begin{array}{c}\mathrm{D} 23 \\
(\mathrm{~m})\end{array}$ & $\begin{array}{c}\text { D34 } \\
(\mathrm{m})\end{array}$ & $\begin{array}{c}\text { D14 } \\
(\mathrm{m})\end{array}$ & $\begin{array}{c}\text { D13 } \\
(\mathrm{m})\end{array}$ & $\begin{array}{c}\text { D24 } \\
(\mathrm{m})\end{array}$ \\
\hline Ref. & 63.216 & 50.480 & 70.636 & 62.167 & 87.560 & 86.821 \\
\hline UAV & 63.164 & 50.499 & 70.637 & 62.114 & 87.525 & 86.796 \\
\hline error & 0.052 & -0.019 & -0.001 & 0.053 & 0.035 & 0.025 \\
\hline
\end{tabular}

Table 2. Check distances (merged blocks)

Comparing Table 1 and Table 2 reveals a significant improvement in the result that verifies our hypothesis for the possibility of using local low altitude flights with larger external accuracy information, in this case RTK-GNSS, to enhance the photogrammetric block scale estimation. In addition to setup 1 and setup 2, we also investigated three other different setups which are listed in Table 3 as well.

\begin{tabular}{|l|c|}
\hline \multicolumn{1}{|c|}{ Setup } & $\begin{array}{c}\text { error (m) on } \\
\text { check distances }\end{array}$ \\
\hline Setup 1: Main block (55m alt., No RTK) & $2.064 \pm 0.901$ \\
\hline $\begin{array}{l}\text { Setup 2: Setup 1 + small blocks in the } \\
\text { corners (10 m alt. with RTK) }\end{array}$ & $0.024 \pm 0.027$ \\
\hline $\begin{array}{l}\text { Setup 3: Setup 1 + small blocks in the } \\
\text { corners (10 m alt., NO RTK) }\end{array}$ & $2.635 \pm 0.507$ \\
\hline $\begin{array}{l}\text { Setup 4: Setup 3 + three scale-bars in } \\
\text { each small block (NO RTK) }\end{array}$ & $-0.035 \pm 0.017$ \\
\hline $\begin{array}{l}\text { Setup 5: Setup 2 + three scale-bars in } \\
\text { each small block (with RTK) }\end{array}$ & $0.018 \pm 0.019$ \\
\hline
\end{tabular}

Table 3. Results of different setups in the first experiment 
All the error values in Table 3 are distance errors and using error propagation, the precision of 3D coordinates could be estimated via dividing the precision of distances by the factor of $\sqrt{2}$.

We can summarize the main findings of as follows:

- Setup 1: using images with very low accurate camera positions $(10 \mathrm{~m} \pm 2 \mathrm{~m})$ and without GCPs leads to very low accurate $(2.1 \pm 0.9 \mathrm{~m})$ local distance measurements

- Setup 2: availability of the RTK information for small low altitude blocks (without using scale-bars) can expressively improves the results.

- Setup 3: Adding small low altitude blocks in the corners of the scene without RTK information cannot improve the accuracy.

- Setup 4: Adding scale-bars to setup 3 improves the result significantly $(-3.5 \pm 1.7$ centimeters $)$.

- Setup 5: Merging images from small low altitude blocks with RTK and employing scale-bars in those small areas provides the best result ( $1.8 \pm 1.9$ centimeters).

Both solutions (adding low altitude flights or adding scale-bars) improved the results significantly from some meters to some centimeters. Integrating both solutions provided the best results.

It worth mentioning that, in this experiment we just used two short parallel strips for each local low altitude flight and this could be a reason that we could not get even better results. Therefore, in the second experiment we use a grid pattern for the flight over small corner areas at the low altitude and will investigate the possible improvements.

\subsection{Second experiment}

In the second experiment 171 images at $55 \mathrm{~m}$ altitude and 310 images (in average) from each of four small corners at $10 \mathrm{~m}$ height are captured (Figure $4 \mathrm{a}$ and $\mathrm{b}$ ). RTK information of all images are available and used in solving the photogrammetric blocks.

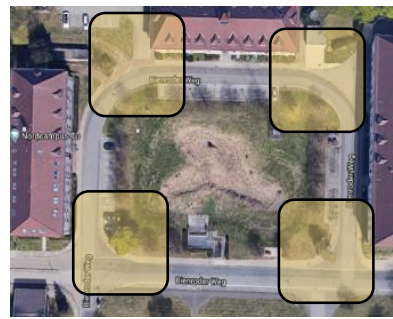

a

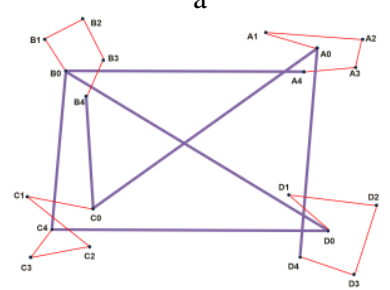

c

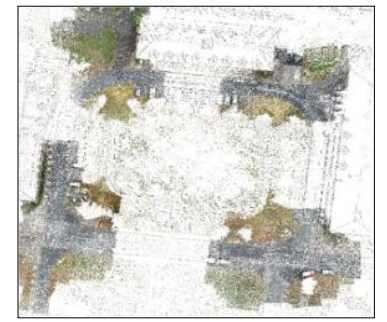

b

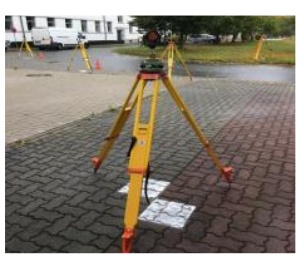

d
Figure 4: Configuration of second experiment; a) Study area which captured from $55 \mathrm{~m}$ height and 4 small areas captured from $10 \mathrm{~m}$ height, b) sparse point cloud of merged flights, c) check distances which are employed for analyzing the results, d) surveying setup at one of the small corner areas.
The whole study area is captured from $55 \mathrm{~m}$ height. In addition, small corner areas are captured at the flight height of $10 \mathrm{~m}$ separately (Figure 4b). In each area three scale-bars are spread on the grounds. The average GSD of images at $10 \mathrm{~m}$ and $55 \mathrm{~m}$ altitudes are $0.27 \mathrm{~cm}$ and $1.62 \mathrm{~cm}$, respectively. Markers on the scale-bars are not measurable (and even visible) in $55 \mathrm{~m}$ altitude images (Figure 5a). However, lower GSD of local $10 \mathrm{~m}$ flight allows the automatic measurement of the markers installed on the scale-bars (Figure 5b).

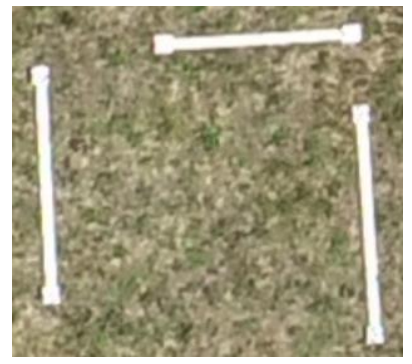

a

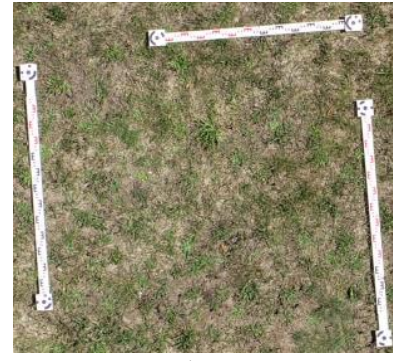

Figure 5: Sample scale-bars on 55m and 10m altitude flights.

On each corner of the study area, a bunch of five points are measured using a MS50 Leica multi station. Similar to our first experiment these points serve as check points for computing ground truth distances and are not used in the block adjustment computations. Moreover, all four bunches of check points (one in each corner of the test area) are parts of a bigger closed surveying network with a standard deviation in XYZ of $1 \mathrm{~mm}$ to make a global network adjustment.

For this experiment, 27 check distances (Figure 4c) are measured in 3 different setups (Table 4).

\begin{tabular}{|l|}
\hline Setup 1: Main block (55m altitude) \\
\hline Setup 2: Setup 1 + small blocks in the corners (10 m altitude) \\
\hline Setup 3: Setup 2 + three scale-bars in each small block \\
\hline
\end{tabular}

Table 4. Various setups in the second experiment

By comparing the ground truth distances (surveying measurements) with measurements on each of the setups, the errors are computed and are reported in Figure 6.

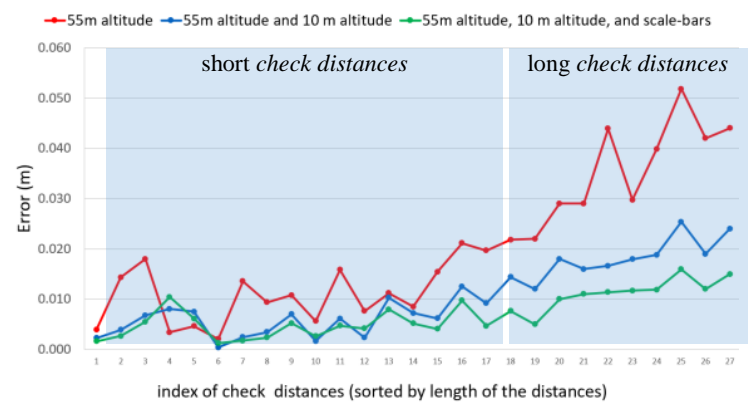

Figure 6: Error on check distances. Short distances are the distances within small areas (captured also at $10 \mathrm{~m}$ altitude), and long distances means the distances between block corners.

The dominant trend in Figure 6 shows the fact that for almost all check distances, adding the images with lower altitude flights improves the results and adding scale-bars improves the results of the combined project even more. Using the project which 
contains just the images from $55 \mathrm{~m}$ height, the maximum error is more than $5 \mathrm{~cm}$. However, merging the project of $10 \mathrm{~m}$ height images, the maximum error is $2.5 \mathrm{~cm}$ which shows the $50 \%$ improvement. And adding scale-bars the maximum error of $1.7 \mathrm{~cm}$ is achieved. Figure 7 illustrates the box plot of the errors on check distances for all three setups.

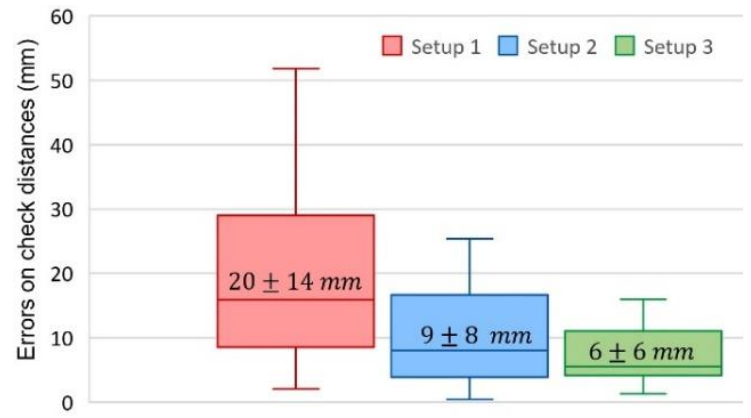

Figure 7: Error on check distances.

By comparing Setup 1 and Setup 2 in the Figure 7, it is clear that adding low altitude flights improves all the quality metrics (maxmin error, mean error, and standard deviation of the errors) in our experiment. Setup 3 in Figure 7 also reveals that by adding scalebars further improvement on all quality metrics is achieved.

Comparing the length of the ground truth distances with imagebased distances just using the images from $55 \mathrm{~m}$ with RTK information (Setup 1), the RMSE of the check distances is 2.4 $\mathrm{cm}$. Adding the projects with $10 \mathrm{~m}$ altitude (Setup 2) decreases the accuracy of the check distances to $1.2 \mathrm{~cm}$. Furthermore, adding scale-bars improves the RMSE of distances to $0.8 \mathrm{~cm}$. This means using low altitude flight and calibrated scale-bar $50 \%$ and $67 \%$ internal accuracy improvement is achieved. This reveals that adding low-altitude flight in a small part of the block and also employing scale-bars improves the quality of scale estimation in order to fully employ the image contents.

In order to remove the effect of short distances on the results and having more realistic estimations for long distances, in the next step just the long check distances between corner area (ranging from $44 \mathrm{~m}$ to $100 \mathrm{~m}$ ) are considered. In this evaluation setup, using the images from $55 \mathrm{~m}$ with RTK information, the RMSE of the check distances is $3.5 \mathrm{~cm}$. Merging the projects with $10 \mathrm{~m}$ altitude decreases the RMSE of the long check distances to 1.8 $\mathrm{cm}$ and adding scale-bars improves the RMSE of distances to 1.1 $\mathrm{cm}$. As expected, the results of using all check distances was very optimistic and better than this evaluation setup. However, using long check distances gives a more reliable estimation of the error throughout the whole project for long distances.

Fitting a model for predicting the error: It could be understood from best results in Figure 6 Setup 3 that there is a quite linear relation between the length of the check distances and their errors. Therefore, we used half of the check distances to train a linear regression model for fitting a linear model (Figure 8) and predicting the error of distances. The computed linear model is as follows:

$$
e= \pm(3+0.1 D) \mathrm{mm}
$$

where $D$ is a distance (in meters) and $e$ is its estimated error in millimeters. In order to validate the model, we used the remaining half of the distances as test set and computed the discrepancy of the image-based length measurements with respect to the ground truth (accurate surveying distances). The mean error on this test set is $1.5 \mathrm{~mm}$ and standard deviation of errors is $1.2 \mathrm{~mm}$. It means that using our setup for this experiment and employing our model for predicting the error, the expected error of distances would be reliable in range of $\pm 3 \mathrm{~mm}$ in $68 \%$ confidence interval and $5 \mathrm{~mm}$ in $99 \%$ confidence interval.

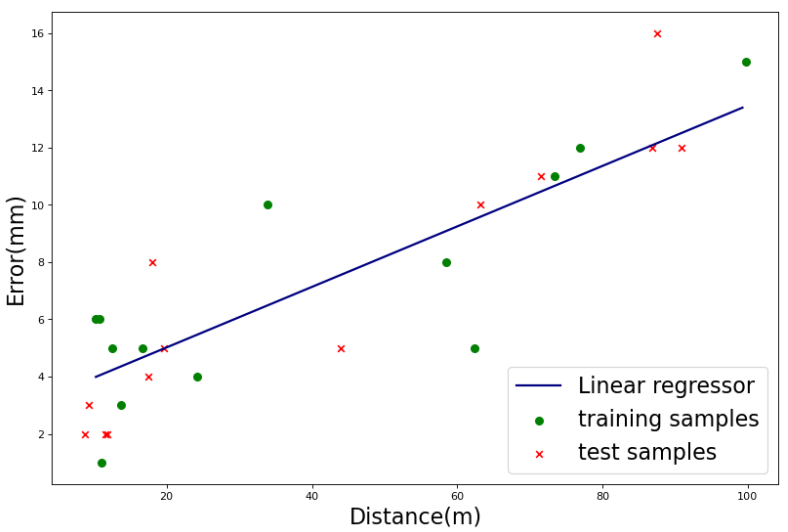

Figure 8: Fitting a linear model for predicting the error of local measurements

It should be accentuated that we verified our model for the distances in range of $9 \mathrm{~m}$ to $100 \mathrm{~m}$ which could be sufficient for local measurements in many applications. Nevertheless, this model could not be generalized for distances out of this range without further investigation.

Using less low altitude flights and scale-bars: Promising result of Setup 3 in our second experiment (adding 4 low altitude flight with 3 scale-bars in each of them), motivated more investigations to analyze the effect of number of low altitude flights and number of scale-bars on the accuracy of the local measurements. To this end, we decrease the number of low altitude flights of Setup 3 to one and two to check the possible decrease in the accuracy of the check distances

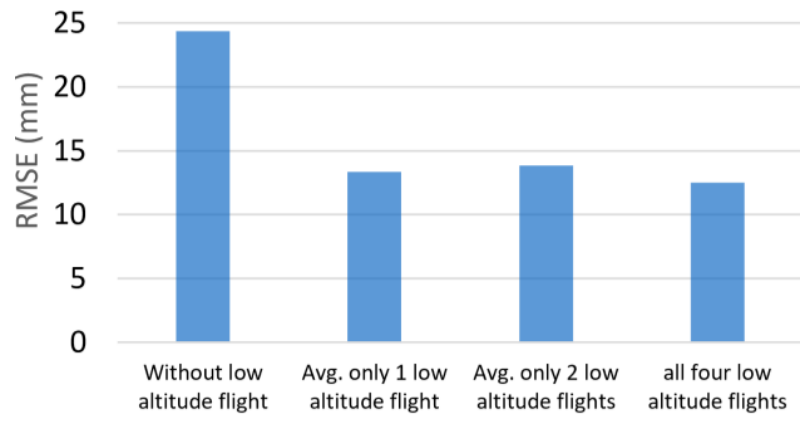

Figure 9: Analyzing the effect of number of low altitude flights on errors

Interestingly, our investigation reveals that using just one or two low altitude flights gives quite similar average results as using all four low altitude flights. Using just one low altitude flight, the average RMSE on check distances is $13 \mathrm{~mm}$ and using two low altitude flights results in $14 \mathrm{~mm}$ RMSE and using all four low altitude flights over four corners of the project leads to RMSE of $12 \mathrm{~mm}$. The very slight changes may originate from measurement errors and are neglectable. This means that instead of having four low altitude flights, less low altitude flights may bring the similar improvement in the result. This decreases the effort, time and cost comparing to four corner flights. However, flying over two cross corners of the project may be more reliable in practice. In case of using just one low altitude flight in our experiments, we got the RMSE values of $14,11,9$, and $19 \mathrm{~mm}$. Although the average RMSE is $13 \mathrm{~mm}$, the RMSE differences makes this setup less reliable. 
In our final investigation, instead of 3 scale-bars in each small area (low altitude flights) we used just 1 scale-bar in each area and computed the errors. In this investigation we compared the distances of 27 distances with ground truth (accurate geodetic measurements) and the results show that in both cases we can achieve similar result. In both cases, RMSE $=8 \mathrm{~mm}$ and bias $=$ $6 \mathrm{~mm}$. This means that even just using one scale-bar in each corner of the study area, scale estimation gets much more accurate and RMSE value is decreased from $24 \mathrm{~mm}$ to $8 \mathrm{~mm}$ which is equivalent to $67 \%$ improvement in local measurements. Comparing also long check distances (11 out of 27 distances) which are longer than $44 \mathrm{~m}$, RMSE value of $11 \mathrm{~mm}$ is achieved. Without local flights and scale-bars this value was $35 \mathrm{~mm}$. This verifies similar accuracy improvement even for long distances.

\section{CONCLUSUION}

In various projects, local measurements (e.g. distances, and volumes) are of high interest. We proposed a strategy to improve the internal accuracy of UAV image blocks to increase the accuracy of local image-based measurements. Adding local lowaltitude flight(s) and utilizing accurate scale-bars as scale constraint in block adjustment are used to facilitate the accuracy improvement without using GCPs. In our first experiment a scenario without RTK information for camera position is exploited. Our proposed setup leads to increasing the accuracy of distance measurements from 1-3 $\mathrm{m}$ to less than 4 centimeters, which is still not exploiting the full potential of the low image flight which has a nominal GSD of about $3 \mathrm{~mm}$. In the second experiment where RTK-GNSS is available, various setups for improving the internal accuracy of images block are investigated. 27 check distances are used as ground truth to analyze the results. While the RMSE of the main block with $55 \mathrm{~m}$ altitude is $2.4 \mathrm{~cm}$, adding low altitude flights decreased the RMSE to $1.2 \mathrm{~cm}$ and adding scale-bars to the merged flights yields RMSE value of 0.8 $\mathrm{cm}$ on the check distances.

Our investigations also show that similar accuracy level using just one local low-altitude flight is achievable. However, flying over two cross corners of the project is more reliable in practice. Our analysis also reveals that using just one scale-bar in each area with low altitude flight provides the similar results as using three scale-bars in each area. In fact, for small area projects we could show that the possible internal measurement accuracy, traditionally related by the GSD can be propagated into the block using a couple of local scale constraints: over the whole block, distance measurements are up to an averaged GSD accurate. However, using those simple slant distances, block deformation cannot be detected or mitigated. It might therefore be necessary to analyze those in more detail, specifically by adding more vertical reference data and by enlarging the project area.

\section{REFERENCES}

Adjidjonu, D., Burgett, J., 2021. Assessing the Accuracy of Unmanned Aerial Vehicles Photogrammetric Survey. Int. J. Constr. Educ. Res. 17, 85-96. https://doi.org/10.1080/15578771.2020.1717683

Burdziakowski, P., 2020. Increasing the Geometrical and Interpretation Quality of Unmanned Aerial Vehicle Photogrammetry Products using Super-Resolution Algorithms. Remote Sens. 12, 810. https://doi.org/10.3390/rs12050810

Ekaso, D., Nex, F., Kerle, N., 2020. Accuracy assessment of realtime kinematics (RTK) measurements on unmanned aerial vehicles (UAV) for direct geo-referencing. Geo-spatial Inf. Sci. $23,165-181$.
Ferrer-González, E., Agüera-Vega, F., Carvajal-Ramírez, F., Martínez-Carricondo, P., 2020. UAV photogrammetry accuracy assessment for corridor mapping based on the number and distribution of ground control points. Remote Sens. 12.

Gerke, M., Nyaruhuma, A.P., 2009. Incorporating scene constraints into the triangulation of airborne oblique images, in: High-Resolution Earth Imaging for Geospatial Information: ISPRS XXXVIII 1-4-7/WS. International Society for Photogrammetry and Remote Sensing (ISPRS).

Gerke, M., Przybilla, H.J., 2016. Accuracy analysis of photogrammetric UAV image blocks: Influence of onboard RTK-GNSS and cross flight patterns. Photogramm. $\begin{array}{llr}\text { Fernerkundung, } & \text { Geoinf. } & \text { 2016, }\end{array}$ https://doi.org/10.1127/pfg/2016/0284

Ghassoun, Y., Gerke, M., Khedar, Y., Backhaus, J., Bobbe, M., Meissner, H., Tiwary, P.K., Heyen, R., 2021. Implementation and Validation of a High Accuracy UAV-Photogrammetry Based Rail Track Inspection System. Remote Sens. 13, 384. https://doi.org/10.3390/rs13030384

González, D., Patricio, M.A., Berlanga, A., Molina, J.M., 2019. A super-resolution enhancement of UAV images based on a convolutional neural network for mobile devices. Pers. Ubiquitous Comput. 1-12. https://doi.org/10.1007/s00779-01901355-5

Kedzierski, M., Wierzbicki, D., 2016. Methodology of improvement of radiometric quality of images acquired from low altitudes. Meas. J. Int. Meas. Confed. 92, 70-78. https://doi.org/10.1016/j.measurement.2016.06.003

Kraft, T., Geßner, M., Meißner, H., Cramer, M., Gerke, M., Przybilla, H.J., 2016. Evaluation of a Metric Camera System Tailored for High Precision UAV Applications, in: IAPRS, Vol. XLI-B1. pp. 901-907. https://doi.org/10.5194/isprs-archives-xlib1-901-2016

Malihi, S., ValadanZoej, M.J., Hahn, M., 2018. Large-Scale Accurate Reconstruction of Buildings Employing Point Clouds Generated from UAV Imagery. Remote Sens. 10, 1148. https://doi.org/10.3390/RS10071148

Manfreda, S., Dvorak, P., Mullerova, J., Herban, S., Vuono, P., Arranz Justel, J., Perks, M., 2019. Assessing the Accuracy of Digital Surface Models Derived from Optical Imagery Acquired with Unmanned Aerial Systems. Drones 3, 15. https://doi.org/10.3390/drones3010015

Martínez-Carricondo, P., Agüera-Vega, F., Carvajal-Ramírez, F., Mesas-Carrascosa, F.J., García-Ferrer, A., Pérez-Porras, F.J., 2018. Assessment of UAV-photogrammetric mapping accuracy based on variation of ground control points. Int. J. Appl. Earth Obs. Geoinf. 72, 1-10. https://doi.org/10.1016/j.jag.2018.05.015

Przybilla, H.J., Gerke, M., Dikhoff, I., Ghassoun, Y., 2019. Investigations on the geometric quality of cameras for uav applications using the high precision uav test field zollern colliery, in: IAPRS. pp. 531-538. https://doi.org/10.5194/isprsarchives-XLII-2-W13-531-2019

Sahebdivani, S., Arefi, H., Maboudi, M., 2020. Rail Track Detection and Projection-Based 3D Modeling from UAV Point Cloud. Sensors 20, 5220. https://doi.org/10.3390/s20185220

Sanz-Ablanedo, E., Chandler, J., Rodríguez-Pérez, J., Ordóñez, C., 2018. Accuracy of Unmanned Aerial Vehicle (UAV) and SfM Photogrammetry Survey as a Function of the Number and Location of Ground Control Points Used. Remote Sens. 10, 1606. https://doi.org/10.3390/rs10101606 